\title{
The surface impedance of noble metals in the near infrared. A new approximation of the Maxwell-Boltzmann equation
}

\author{
J. C. Dudek \\ CNAM, 292, rue Saint-Martin, 75141 Paris Cedex 03, France
}

(Reçu le 12 octobre 1984, révisé les 12 mars et 25 avril 1986, accepté le 15 mai 1986)

\begin{abstract}
Résumé. - En partant de la résolution numérique des équations semi-classiques de l'effet de peau anormal, nous montrons que le couplage électromagnétique entre électrons de conduction devient totalement négligeable dans le domaine des faibles longueurs d'ondes $(\lambda<2 \mu \mathrm{m}$ pour les métaux nobles), ce qui entraîne d'importantes simplifications des algorithmes permettant le calcul de l'impédance de surface. En outre, les résultats du calcul numérique montrent que ces algorithmes simplifiés restent encore utilisables jusque vers $\lambda=20 \mu \mathrm{m}$, dans un domaine de longueur d'onde où le couplage électron-électron est faible mais non négligeable. La forme simplifiée de l'équation de Maxwell-Boltzmann qui en découle constitue donc une nouvelle approximation de la théorịe semi-classique, valable dans le domaine d'un faible effet de peau anormal.
\end{abstract}

\begin{abstract}
From the exact numerical resolution of the discretized semi-classical equations of the anomalous skin effect theory, we prove that E.M. electron-electron coupling vanishes in the limit of short wavelengths ( $\lambda$ typically shorter than $2 \mu \mathrm{m}$ for noble metals). That leads to substantial simplification of the algorithms allowing the calculation of the surface impedance. In addition, numerical results show that these simplified algorithms remain very accurate up to wavelengths about $20 \mu$ where E.M. e-e coupling is weak but not negligible. We conclude that the corresponding simplified form of the Maxwell-Boltzmann equation constitutes a new approximation of the semiclassical theory, valid in the weak anomalous skin effect regime.
\end{abstract}

\section{Introduction.}

In a preceding paper [1], we have presented a new method for computing exactly the surface impedance $Z_{\mathrm{s}}$ of a semi-infinite metal for any value of the Fuchs scattering parameter $p$. This method lies on a discretization of Reuter and Sondheimer semi-classical equations [2].

The macroscopic E.M. fields are expressed as sums of coupled partial fields, each of them being associated with one discrete value of the electrons normal momentum.

In the spherical approximation, a given normal momentum value on the Fermi surface will correspond to one angle $\theta$ between the momentum vector and the metal surface; consequently, anisotropy $p(\theta)$ of the surface scattering parameter and $\tau(\theta)$ of the relaxation time can easily be introduced in the discrete equations. The accuracy of the calculations increases very quickly with the number $N$ of discrete components involved. It has been shown that values of $N$ as small as 4 give agreement with Dingle's series [3] better than $3 \%$ in the wavelength domain [1-200 $\mu \mathrm{m}]$.
Unfortunately, the implementation of this method on a digital computer becomes very difficult when anisotropy must be precisely taken into account or (and) when it is applied to thin films because, in both cases, great $N$ values are required and numerical overflows occur in the coefficients of the characteristic equation. In addition, this latter must be solved by means of an iterative method which becomes very timeconsuming for large $N$.

However, examination of the non-local part of the electric field matrix shows that the non-diagonal components become negligible at short wavelengths $(\lambda<2 \mu \mathrm{m})$. That may be interpreted physically as being due to the vanishing of E.M. electron-electron coupling and the electric field may be expressed as the sum of $N$ independent two-wave partial fields attached respectively to $N$ sets of electrons moving at discrete angles $\theta_{n}$.

This result is derived rigorously in the high frequency limit, in section 2.

It is shown in section 3 that the high frequency expression of the field implies that the basic equations of the anomalous skin effect may be solved indepen- 
dently for each conduction electron; i.e., the perturbation of the Fermi surface in a given direction $\theta$ depends of the only two-wave field component attached to the electrons moving in this direction.

In this simplified approach, the Maxwell-Boltzmann propagation equation and the boundary conditions are rewritten under the form of $N$ independent systems giving $N$ partial admittances $Y_{n}$ the sum of which is identical, at high frequencies, with the reciprocal surface impedance computed by using the exact method.

The numerical results of section 4 indicate that the uncoupled electrons model is more accurate than the exact one in the weak anomalous skin effect domain (relative precision of $10^{-3}$ at $\lambda<20 \mu \mathrm{m}$ ).

This accuracy is not only due to the great value of $N$ accepted by the algorithms but also to the fact that the uncoupled electrons approach is valid as long as the frequency $f$ is such that the dimensionless quantity $\left(\frac{v_{\mathrm{F}}}{c} \frac{f_{0}}{f}\right)^{2}$ is smaller than unity ( $f_{0}$ is the plasma frequency). Moreover, anisotropies may be taken into account more precisely, particularly in thin films for which a too small value of $N$ originates short period calculus oscillations (a few angstroms) in the curves of $Z_{\mathrm{s}}$ versus thickness.

\section{High frequency limit of the electric field matrix.}

2.1 Exact CAlCUlation OF THE ELECTRIC FIELD MATRIX COEFFICIENTS. - In this subsection are used the notations and results of reference [1] where more details will be found.

The total electric field amplitude space variation may be expressed as :

$$
E(z)=\sum_{n=1}^{N} \sum_{p=0}^{N} a_{n p} \mathrm{e}^{i u_{p} z} .
$$

It must be noticed that the subscript $p$ is varied from 0 to $N$ instead of 1 to $N+1$ as in reference [1].

Subscript 0 will be used later to distinguish the quasi-classical components $a_{n 0} \exp \left(i u_{0} z\right)$ from the others which will be qualified as «non-local» components.

The metal is supposed to occupy the half space $z>0$ and its surface lies in the plane $z=0$.

The E.M. fields are all time-dependent by the same factor $\exp (-i \omega t)$.

The $u_{p}$ are eigenwavevectors solutions of the following bulk characteristic equation :

$$
\sum_{n=1}^{N} \frac{B_{n}}{\left(u_{p}^{2}+\alpha_{n}^{2}\right)\left(u_{p}^{2}-k_{r}^{2}\right)}=1
$$

with :

$$
B_{n}=\frac{3}{2} i \omega \mu_{0} \frac{\sigma}{l} \frac{1-s_{n}^{2}}{s_{n}} \alpha_{n} \Delta_{n}
$$

$s_{n}=\sin \theta_{n}(n=1$ to $N)$ is the discrete variable which corresponds to the electrons velocity discrete angle $\theta_{n}$ with respect to the surface.

$\Delta_{n}$ is the interval around $s_{n}\left({ }^{1}\right)$,

$\sigma$ and $l$ are respectively the bulk conductivity and the conduction electrons mean free path.

We have put :

$$
\alpha_{n}=\frac{1-i \omega \tau}{l s_{n}} \text { where } \tau=\frac{l}{v_{F}} .
$$

The Fermi velocity $v_{\mathrm{F}}$ and the ratio $\sigma / l$ are intrinsic constants of the metal that one needs for numerical computations.

$\mu_{0}$ is the vacuum magnetic permeability.

$k_{\mathrm{r}}^{2}=\varepsilon_{\mathrm{r}}\left(\frac{\omega}{c}\right)^{2}$ where $\varepsilon_{\mathrm{r}}$ is the local dielectric constant of the metallic ions plus vacuum background.

There have been defined also $N+1$ partial amplitudes :

$$
a_{p}=\sum_{n=1}^{N} a_{n p} \quad p=0 \text { to } N
$$

which are solutions of the following boundary equations system written for a metal of semi-infinite extent :

$$
\begin{gathered}
\sum_{p=0}^{N} a_{p}=1 \\
\sum_{p=0}^{N} \frac{\left(1-p_{n}\right) \alpha_{n}-\left(1+p_{n}\right) i u_{p}}{\alpha_{n}^{2}+u_{p}^{2}} a_{p}=0 \quad n=1 \text { to } N
\end{gathered}
$$

(4a) comes from the assumption that $E(z=0)=1$.

$p_{n}$ is the Fuchs surface scattering parameter in the direction $\theta_{n}$.

The electric field matrix coefficients will be finally expressed by :

$$
a_{n p}=\frac{B_{n}}{\left(u_{p}^{2}+\alpha_{n}^{2}\right)\left(u_{p}^{2}-k_{\mathrm{r}}^{2}\right)} a_{p} .
$$

2.2 High FREQUENCY LIMIT OF THE EIGENWAVEVECTORS. - The characteristic equation (2) may be slightly modified as :

$$
\sum_{n=1}^{N} \frac{\beta_{n}}{\left(1+\frac{u_{p}^{2}}{\alpha_{n}^{2}}\right) \frac{u_{p}^{2}}{\alpha_{n}^{2}}}=1-\frac{k_{r}^{2}}{u_{p}^{2}}
$$

in which appears the dimensionless parameter :

$$
\beta_{n}=\frac{B_{n}}{\alpha_{n}^{4}}=\frac{3}{2}\left(1-s_{n}^{2}\right) \Delta_{n} \frac{i \sigma \omega \mu_{0}}{l s_{n}} \frac{1}{\alpha_{n}^{3}} .
$$

( $\left.{ }^{1}\right)$ It can be shown that the ratio $Y_{n} / \Delta_{n}$ in which $Y_{n}$ is the partial surface admittance defined in subsection 2.3 , tends to a finite limit as $s_{n} \rightarrow 0$. This is in agreement with the shape of the electron effectiveness curves presented in subsection 4.2. These latters vary smoothly in the vicinity of $\theta=0$ (grazing angles), which allows one to use finite intervals $\Delta_{n}$ for the grazing electrons. 
In the relaxation region as defined by Holstein [4], we have $\omega \tau \gg 1$ and thus :

$$
\left|\beta_{n}\right| \approx \frac{3}{2}\left(1-s_{n}^{2}\right) s_{n}^{2}\left(\frac{v_{\mathrm{F}}}{c} \frac{\omega_{0}}{\omega}\right)^{2} \Delta_{n}
$$

where $\omega_{0}^{2}=n_{\mathrm{e}} e^{2} / m^{*} \varepsilon_{0}$ is the square of the bulk plasmon angular frequency.

In the near infrared (say vacuum wavelength about $1 \mu \mathrm{m}$ ), we have typically for noble metals :

$$
\left(\frac{v_{\mathrm{F}}}{c} \frac{\omega_{0}}{\omega}\right)^{2} \approx 2.5 \times 10^{-3} \text {. }
$$

Therefore, we shall consider that inequality $\left|\beta_{n}\right| \ll \Delta_{n}$ holds for all $n$. The characteristic equation (6) will give then $N$ solutions :

$$
u_{p} \approx i \alpha_{p} \quad p=1 \text { to } N
$$

and one solution $u_{0}$ such that the condition $\left|\frac{u_{0}}{\alpha_{n}}\right|^{2} \ll 1$ is fulfilled for all $n$. It will be verified $a$ posteriori that $\left|\frac{k_{\mathrm{r}}}{u_{0}}\right|^{2} \ll 1$ if $\left(\frac{\omega}{\omega_{0}}\right)^{2} \ll 1$; thus, equation (6) may be written as :

$$
u_{0}^{2} \#\left(1+\frac{k_{\mathrm{r}}^{2}}{u_{0}^{2}}-\sum_{n=1}^{N} \beta_{n}\right) \sum_{n=1}^{N} \beta_{n} \alpha_{n}^{2}
$$

or :

$$
\begin{aligned}
u_{0}^{2} \#-\left(\frac{\omega_{0}}{c}\right)^{2}\left(1+\frac{i}{\omega \tau}\right)^{-1}(1+ & \left.\frac{k_{\mathrm{r}}^{2}}{u_{0}^{2}}-\sum_{n=1}^{N} \beta_{n}\right) \times \\
& \times \sum_{n=1}^{N} \frac{3}{2}\left(1-s_{n}^{2}\right) \Delta_{n} .
\end{aligned}
$$

Use has been made of the relation :

$$
\omega_{0}^{2}=\frac{\sigma}{\tau \varepsilon_{0}} .
$$

If $N$ is a sufficiently large number, we have :

$$
\sum_{n=1}^{N} \frac{3}{2}\left(1-s_{n}^{2}\right) \Delta_{n} \# 1 \text {. }
$$

For example, if the intervals $\Delta_{n}$ are taken equal to $1 / N$ and if $N=4$, this relation is verified with an accuracy of 0.008 . In the high frequency limit, the $\operatorname{sum} \sum_{n} \beta_{n}$ may be neglected and one obtains :

$$
u_{0} \# u_{c l}=\left[k_{\mathrm{r}}^{2}-\left(\frac{\omega_{0}}{c}\right)^{2} \frac{1}{1+\frac{i}{\omega \tau}}\right]^{1 / 2} \text {. }
$$

This expression is consistent with the preceding hypothesis according to which $\left|\frac{k_{\mathrm{r}}}{u_{0}}\right|^{2} \ll 1$. It is exactly the result given by the classical Drude theory.
The non classical solutions (9) may be expressed more precisely in the limit $\left|\beta_{n}\right| \rightarrow 0$ since the characteristic equation (6) simplifies to :

$$
\frac{\beta_{p}}{\left(1+\frac{u_{p}^{2}}{\alpha_{p}^{2}}\right) \frac{u_{p}^{2}}{\alpha_{p}^{2}}} \# 1 \quad p=1 \text { to } N \text {. }
$$

Its solution developed to first order in the parameter $\beta_{p}$ is :

$$
u_{p} \# i \alpha_{p}\left(1+\frac{\beta_{p}}{2}\right) \quad p=1 \text { to } N .
$$

\subsection{APPROXIMATE EXPRESSION OF THE ELECTRIC FIELD} MATRIX. - Expression (1) may be written in matricial form as :

$$
[E]=\left[\begin{array}{c}
E_{1} \\
E_{2} \\
\vdots \\
E_{N}
\end{array}\right]=[A]\left[\begin{array}{c}
W_{0} \\
W_{1} \\
\vdots \\
W_{N}
\end{array}\right]
$$

The $E_{n}$ are the partial waves $\sum_{p=0}^{N} a_{n p} \mathrm{e}^{i u_{p} z}$ associated with all electrons the velocity of which makes the angle $\theta_{n}$ with respect to the surface.

The amplitude matrix [A] is composed of the $a_{n p}$ whereas the $W_{p}(p=0$ to $N)$ are the bulk eigenwaves $\exp \left(i u_{p} z\right)$ among which $W_{0}$ represents the quasiclassical one.

The reciprocal surface impedance is given [1] by :

$$
\frac{1}{Z_{s}}=\frac{1}{\omega \mu_{0}} \sum_{n=1}^{N} \sum_{p=0}^{N} a_{n p} u_{p}
$$

from which $N$ partial surface admittances $Y_{n}$ may be defined by :

$$
\frac{1}{Z_{\mathrm{s}}}=\sum_{n=1}^{N} Y_{n}
$$

and :

$$
\left[\begin{array}{c}
Y_{1} \\
Y_{2} \\
\vdots \\
Y_{N}
\end{array}\right]=\frac{1}{\omega \mu_{0}}[A]\left[\begin{array}{c}
u_{0} \\
u_{1} \\
\vdots \\
u_{N}
\end{array}\right]
$$

After (5), it is obvious that in the relaxation region where $u_{p} \# i \alpha_{p}(p \neq 0)$, the $a_{n p}(n \neq p)$ may be neglected compared with the $a_{p p}(p \neq 0)$.

The field amplitude matrix will be consequently approximated by :

$$
[A]=\left[\begin{array}{cccccc}
a_{10} & a_{11} & 0 & \ldots & 0 \\
a_{20} & 0 & a_{22} & \ldots & 0 \\
\vdots & \vdots & \vdots & \ddots & \\
a_{N_{0}} & 0 & 0 & \ldots & a_{N N}
\end{array}\right]
$$


It has been shown in reference [1] (see also Ref. [20]) that the discrete characteristic equation and boundary conditions will form a self-consistent system only if the number of eigenwaves $\exp \left(i u_{p} z\right)$ is taken equal to $N+1$.

As a given row $n$ of the matrix represents the amplitudes attached to the electrons $s_{n}$, one obtains a rectangular $N \times(N+1)$ matrix.

In the present approximation, the first column contains the classical amplitudes whereas the remaining part may be considered as a purely non local square matrix.

An example of matrix obtained by the exact numerical method is presented in table I for $N=6$.

It confirms that (16) is a very good approximate form for noble metals at wavelengths shorter than $2 \mu \mathrm{m}$.

It should be noticed that the validity of this approximation depends essentially on the smallness of the parameter $\beta_{n}$ which does not depend in turn, after (8), on the electron mean free path i.e. on temperature as long as $\omega \tau \gg 1$.

The matrix coefficients of table I have been calculated in the worst case of completely diffuse surface scattering $\left(p_{n}=0\right.$ for all $\left.n\right)$.

When surface scattering is partially or completely specular, the non diagonal components of the non local part of $[A]$ are found to be smaller and thus, the above approximation better.

2.4. QUASI-CLASSICAL AND NON LOCAL FIELD AMPLITUDES. - In the high frequency limit, the validity of the approximate form (16) for the electric field matrix implies that the boundary equations (4b) may be simplified as :

$$
\begin{aligned}
& \frac{\left(1-p_{n}\right) \alpha_{n}-\left(1+p_{n}\right) i u_{0}}{\alpha_{n}^{2}+u_{0}^{2}} a_{0}+ \\
& +\frac{\left(1-p_{n}\right) \alpha_{n}-\left(1+p_{n}\right) i u_{n}}{\alpha_{n}^{2}+u_{n}^{2}} a_{n n} \# 0, n=1 \text { to } N .
\end{aligned}
$$

We shall use in the following the same inequalities as in subsection 2.2 i.e. :

$$
\left|k_{\mathrm{r}}\right|^{2} \ll\left|u_{0}\right|^{2} \ll\left|\alpha_{n}\right|^{2} .
$$

Hence, after equation (13), the coefficients of $a_{0}$ and $a_{n n}$ in (17) will be respectively on the order of $1 / \alpha_{n} \beta_{n}$ and $1 / \alpha_{n}$; thus the ratio $a_{n n} / a_{0}$ will be very small compared with unity.

Moreover, because of definition (3), we have $a_{n} \# a_{n n}$ and the total quasi-classical amplitude will be finally approximated, after equation (4a), by :

$$
a_{0} \# 1-\sum_{n=1}^{N} a_{n n} \# 1 \text {. }
$$

The quasi-classical partial amplitudes will be given, after (5), by :

$$
a_{n_{0}} \# \frac{B_{n}}{\alpha_{n}^{2} u_{0}^{2}}\left(1-\frac{u_{0}^{2}}{\alpha_{n}^{2}}+\frac{k_{\mathrm{r}}^{2}}{u_{0}^{2}}\right) .
$$

In the wavelength domain considered here $(\lambda \approx 1 \mu \mathrm{m})$, one may use the following additional inequality :

$$
\left|\frac{u_{0}}{\alpha_{n}}\right|^{2} \ll\left|\frac{k_{\mathrm{r}}}{u_{0}}\right|^{2} .
$$

Table I. - Electric field matrix components $a_{n p}$ computed with the exact coupled equations (2), (4) and expres-

\begin{tabular}{|c|c|c|c|c|c|c|}
\hline 0 & 1 & 2 & 3 & 4 & 5 & 6 \\
\hline $\begin{array}{r}0.248 E+00 \\
-0.227 E-04\end{array}$ & $\begin{array}{r}0.738 E-0.5 \\
-0.321 E-06\end{array}$ & $\begin{array}{r}-0.942 E-09 \\
0.740 E-09\end{array}$ & $\begin{array}{r}-0.599 E-07 \\
0.374 E-08\end{array}$ & $\begin{array}{r}-0.179 E-06 \\
0.842 E-68\end{array}$ & $\begin{array}{r}-0.321 E-06 \\
0.992 E-08\end{array}$ & $\begin{array}{r}-0.254 E-06 \\
0.357 E-08\end{array}$ \\
\hline $\begin{array}{r}0.234 E+06 \\
-0.157 E-04\end{array}$ & {$\left[\begin{array}{r}0.131 E-10 \\
-0.123 E-11\end{array}\right.$} & $\begin{array}{r}0.631 E-04 \\
-0.173 E-05\end{array}$ & $\begin{array}{r}-0.849 E-07 \\
0.530 E-08\end{array}$ & $\begin{array}{r}-0.203 E-06 \\
0.954 E-68\end{array}$ & $\begin{array}{r}-0.337 E-06 \\
0.104 E-07\end{array}$ & $\begin{array}{r}-0.257 E-\overline{6} 6 \\
\overline{0.361 E-08}\end{array}$ \\
\hline $\begin{array}{r}0.206 E+00 \\
-0.391 E-05\end{array}$ & $\begin{array}{r}0.364 E-11 \\
-0.360 E-12\end{array}$ & $\begin{array}{r}0.392 E-08 \\
-0.308 E-09\end{array}$ & $\begin{array}{r}0.155 E-03 \\
-0.185 E-05\end{array}$ & $\begin{array}{r}-0.298 E-06 \\
0.140 E-07\end{array}$ & $\begin{array}{r}-0.382 \mathrm{E}-06 \\
0.118 \mathrm{E}-07\end{array}$ & $\begin{array}{r}-0.264 E-06 \\
0.371 E-08\end{array}$ \\
\hline $\begin{array}{l}0.164 E+00 \\
\bar{\theta} .877 E-015\end{array}$ & $\begin{array}{r}0.153 E-11 \\
-0.144 E-12\end{array}$ & $\begin{array}{r}0.125 E-08 \\
-0.983 E-10\end{array}$ & $\begin{array}{r}0.398 E-07 \\
-0.248 E-08\end{array}$ & $\begin{array}{l}0.242 E-03 \\
0.862 E-06\end{array}$ & $\begin{array}{r}-0.533 E-66 \\
0.165 E-67\end{array}$ & $\begin{array}{r}-0.281 E-06 \\
0.395 E-08\end{array}$ \\
\hline $\begin{array}{l}0.109 E+00 \\
\text { อ. } 163 E-04\end{array}$ & $\begin{array}{r}0.51 \mathrm{GE}-12 \\
-0.572 \mathrm{E}-13\end{array}$ & $\begin{array}{r}0.461 E-99 \\
-0.362 E-10\end{array}$ & $\begin{array}{r}0.113 E-07 \\
-0.706 E-09\end{array}$ & $\begin{array}{r}0.118 E-06 \\
-0.555 E-08\end{array}$ & $\begin{array}{l}0.264 E-03 \\
0.510 E-05\end{array}$ & $\begin{array}{r}-0.336 E-06 \\
0.473 E-08\end{array}$ \\
\hline $\begin{array}{l}\text { Q. } 395 E-01 \\
\text { B. } 107 E-04\end{array}$ & $\begin{array}{r}0.149 E-12 \\
-0.139 E-13\end{array}$ & $\begin{array}{r}0.198 E-09 \\
-0.850 E-11\end{array}$ & $\begin{array}{r}0.241 E-08 \\
-0.150 E-09\end{array}$ & $\begin{array}{r}0.192 E-07 \\
-0.901 E-09\end{array}$ & $\begin{array}{r}0.103 E-06 \\
-0.318 E-08\end{array}$ & $\begin{array}{l}0.142 E-0.3 \\
0.516 E-05\end{array}$ \\
\hline
\end{tabular}
sion (5) for $N=6, \lambda=2 \mu \mathrm{m}$ and $l=0.1 \mu \mathrm{m}$ in the completely diffuse scattering case $\left(p_{n}=0\right.$ for any $\left.n\right)$. Each component is represented in complex form by its real and imaginary parts (upper and lower numbers respectively). In the first column are the quasi-classical components $a_{n_{0}}$ associated with electrons $s_{n}$. They are far greater than those of the remaining non local part of the matrix.

In addition, the diagonal components in this latter are themselves far greater than the others. 
Then, putting (11) in the above expression of $a_{n_{0}}$, one gets simply :

$$
a_{n_{0}} \# \frac{3}{2}\left(1-s_{n}^{2}\right) \Delta_{n} .
$$

As for the non classical (or non local) components, the only terms $a_{n n} \exp \left(i u_{n} z\right)$ are of importance. Their amplitude can be obtained from the above equations (13) and (17) by putting $a_{0} \# 1$ and using inequalities (18); one gets :

$$
a_{n n} \#\left(1-p_{n}\right) \frac{\beta_{n}}{2}, \quad n=1 \text { to } N
$$

\section{Equivalent set of uncoupled equations.}

3.1. EXPRESSION OF THE SURFACE IMPEDANCE IN THE RELAXATION REGION. - The validity of the preceding formulae can be checked by computing the surface impedance in the limit $\beta_{n} \rightarrow 0$.

The surface admittance may be expressed after (14) as :

$$
Y_{\mathrm{s}} \# \frac{1}{\omega \mu_{0}} \sum_{n=1}^{N}\left(a_{n_{0}} u_{0}+a_{n n} u_{n}\right)
$$

Putting expressions (9), (19) and (20), one gets :

$$
Y_{\mathrm{s}} \# \frac{1}{\omega \mu_{0}}\left[u_{0}+\sum_{n=1}^{N}\left(1-p_{n}\right) i \frac{\beta_{n} \alpha_{n}}{2}\right]
$$

or, after (7) and (11) :

$$
Y_{\mathrm{s}} \# \frac{1}{Z_{c l}}+\frac{1}{Z_{0}} \frac{3}{4} \frac{v_{\mathrm{F}}}{c}\left(\frac{\omega_{0}}{\omega}\right)^{2} \sum_{n=1}^{N}\left(1-s_{n}^{2}\right) s_{n}\left(1-p_{n}\right) \Delta_{n}
$$

with :

$$
\begin{aligned}
& Z_{0}=\left(\frac{\mu_{0}}{\varepsilon_{0}}\right)^{1 / 2}=376.730 \Omega: \text { free space impedance } \\
& Z_{c l}=\frac{Z_{0}}{\sqrt{\varepsilon_{\mathrm{D}}}}: \text { classical surface impedance }
\end{aligned}
$$

and :

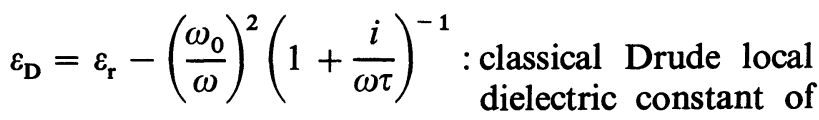
the metal.

The sum over $n$ in (22) may be replaced by the equivalent integral and because of inequality $\omega \tau \gg 1$, the surface impedance expressed as $Z_{\mathrm{s}}=R_{\mathrm{s}}+i X_{\mathrm{s}}$ will be given by :

$$
\begin{aligned}
& R_{\mathrm{s}} \# \frac{Z_{0}}{2 \omega_{0} \tau}\left[1+\frac{3}{8} \frac{\omega_{0}}{c} l(1-\langle p\rangle)\right] \\
& X_{\mathrm{s}} \#-Z_{0} \frac{\omega}{\omega_{0}}\left(1+\frac{\varepsilon_{\mathrm{r}}}{2} \frac{\omega^{2}}{\omega_{0}^{2}}\right)
\end{aligned}
$$

with :

$$
\langle p\rangle=\int_{0}^{1} 4 s\left(1-s^{2}\right) p(s) \mathrm{d} s
$$

$\langle p\rangle$ is an effective equivalent isotropic specularity parameter; its expression (24) is identical to the one given by McKay and Rayne (formula (13) of Ref. [5]).

Expression (23a) of the surface resistance has already been obtained by Holstein [4] in the case of a simple isotropic scattering parameter $p$ and by Dingle [6] in the case of completely diffuse scattering $(\langle p\rangle=0)$. It can be seen after (22) that the additional surface assisted absorption term does not depend on the lectron mean free path i.e. on temperature, in the relaxation region. It may be simply accounted for in the Drude dielectric constant by including it, via Matthiessen's rule, under the form of the following relaxation time [7-9] :

$$
\frac{1}{\tau_{\mathrm{s}}}=\frac{3}{8}(1-\langle p\rangle) \frac{\omega_{0}}{c} v_{\mathrm{F}} .
$$

3.2. Simplified FORM OF THE MAXWELl-BOLtZMANN EQUATION. - The characteristic equation (2) has been derived in reference [1] from the following MaxwellBoltzmann propagation equation :

$$
\begin{array}{r}
{\left[\frac{\partial^{4}}{\partial z^{4}}+\left(k_{\mathrm{r}}^{2}-\alpha^{2}\right) \frac{\partial^{2}}{\partial z^{2}}-k_{\mathrm{r}}^{2} \alpha^{2}\right]} \\
\qquad\left(\begin{array}{c}
\varepsilon \\
\text { or } \\
j
\end{array}\right)=B(s)\left(\begin{array}{c}
E \\
\text { or } \\
J
\end{array}\right)
\end{array}
$$

with :

$$
B(s)=\frac{3}{2} i \omega \mu_{0} \frac{\sigma 1-s^{2}}{l} \alpha \text { and } \alpha=\frac{1-i \omega \tau}{l s}
$$

$s=\sin \theta$ is the continuous form of the preceding discrete variable $s_{n}$.

The total electric field and conduction current are defined by :

$$
E(z)=\int_{0}^{1} \varepsilon(z, s) \mathrm{d} s
$$

and :

$$
J(z)=\int_{0}^{1} j(z, s) \mathrm{d} s
$$

In the relaxation region, the electric field may be approximated by the sum over $n$ of the two-component partial waves :

$$
E_{n} \# a_{n_{0}} \mathrm{e}^{i u_{0} z}+a_{n n} \mathrm{e}^{i u_{n} z}
$$

They may be expressed after (9), (19) and (20) by :

$$
E_{n} \# \frac{3}{2}\left(1-s_{n}^{2}\right) \Delta_{n}\left[\mathrm{e}^{i u_{0} z}+\left(1-p_{n}\right) \frac{\beta_{n}^{*}}{2} \mathrm{e}^{-\alpha_{n} z}\right]
$$


where :

$$
\beta_{n}^{*}=i \frac{\sigma \omega \mu_{0}}{l s_{n}} \frac{1}{\alpha_{n}^{3}}
$$

From a physical point of view, $E_{n}$ is the electric field attached to electrons $s_{n}$. The space variation law of the classical component of wavevector $u_{0}$ is the same for all electrons whereas the non local part is characterized by the phase velocity :

$$
v_{\varphi n}=\frac{\omega}{\mathcal{R e}\left\{i \alpha_{n}\right\}} \approx s_{n} v_{\mathrm{F}} .
$$

This is the normal velocity component of electron $s_{n}$ and thus, the predominance of the diagonal non local components in the electric field matrix may be interpreted as a resonant excitation of the electrons $s_{n}$ by the corresponding non local eigenwaves $\exp \left(-\alpha_{n} z\right)$.

In other words, it seems that when $\beta_{n} / \Delta_{n}$ is very small, the electrons act independently one from each other, at least as for the non local part of the field. Examination of relation (10) shows that this assumption may be considered valid as well as for the quasi-classical part, because we can write :

$$
u_{0}^{2} \# u_{c l}^{2} \sum_{n=1}^{N} \frac{3}{2}\left(1-s_{n}^{2}\right) \Delta_{n} .
$$

The sum contains the discretized form of the usual « effectiveness » function $\frac{3}{2} \cos ^{2} \theta[5]$.

If $N$ is a sufficiently great number, this sum may be taken equal to unity and $u_{0}$ can be retrieved from the simplified characteristic equation :

$$
\frac{\beta_{n}^{*}}{\left(u_{p}^{2}+\alpha_{n}^{2}\right)\left(u_{p}^{2}-k_{\mathrm{r}}^{2}\right)}=1 \quad n=1 \text { to } N
$$

provided that the condition $\left|\beta_{n}^{*}\right| \ll 1$ is fulfilled.

(29) means that if a set $\left(s_{n}-\frac{\Delta_{n}}{2}, s_{n}+\frac{\Delta_{n}}{2}\right)$ of conduction electrons could be considered alone (this is not physically possible because of the Fermi distribution), it would be found that an E.M. wave should propagate under the form of a quasi-classical eigenwave $\exp \left(i u_{0} z\right)\left(u_{0} \# u_{c l}\right)$ plus a narrow non local waves packet practically equivalent to $\exp \left(-\alpha_{n} z\right)$.

Thus, the Maxwell-Boltzmann propagation equation (26) may be simplified as :

$$
\left[\frac{\partial^{4}}{\partial z^{4}}+\left(k_{\mathrm{r}}^{2}-\alpha^{2}\right) \frac{\partial^{2}}{\partial z^{2}}-k_{\mathrm{r}}^{2} \alpha^{2}-B^{*}(s)\right]\left(\begin{array}{c}
\varepsilon \\
\text { or } \\
j
\end{array}\right)=0
$$

where :

$$
B^{*}(s)=i \sigma \omega \mu_{0} \frac{\alpha}{l s}
$$

From a physical point of view, this propagation equation is the limit of equation (26) obtained when E.M. e-e coupling is completely neglected.

The only remaining e-e interactions are then those due to the Pauli exclusion principle, which has been taken into account via the Fermi distribution, and e-e collisions which may be included in an effective bulk relaxation time [10].

3.3. UNCOUPLED BOUNDARY EQUATIONS. - Neglecting E.M. e-e coupling, it is possible to rewrite the boundary equations (4) for each set $s_{n}$ of electrons. One can convince himself that the only way to retrieve the partial wave expression (27) is to transform equations (4) into the $N$ following systems :

$$
\begin{gathered}
a_{n_{0}}^{\prime}+a_{n n}=\frac{3}{2}\left(1-s_{n}^{2}\right) \Delta_{n} \\
\frac{\left(1-p_{n}\right) \alpha_{n}-\left(1+p_{n}\right) i u_{0, n}}{\alpha_{n}^{2}+u_{0, n}^{2}} a_{n 0}+ \\
+\frac{\left(1-p_{n}\right) \alpha_{n}-\left(1+p_{n}\right) i u_{n}}{\alpha_{n}^{2}+u_{n}^{2}} a_{n n}=0
\end{gathered}
$$

with : $n=1$ to $N, u_{0, n}$ represents the quasi-classical eigenwavevector given by (29) for electrons $s_{n}$.

Developed to first order in the parameter $\beta_{n}^{*}, u_{0, n}$ is expressed by :

$$
u_{0, n} \# u_{c l}\left(1-\frac{\beta_{n}^{*}}{2}\right) .
$$

The above equations are valid a priori only if $\beta_{n}^{*} \rightarrow 0$ because we have $u_{0, n} \rightarrow u_{c l}$ for all $n$.

It is then found :

$$
\begin{gathered}
u_{n} \# i \alpha_{n}\left(1+\frac{\beta_{n}^{*}}{2}\right) \# i \alpha_{n} \\
a_{n n} \#\left(1-p_{n}\right) \frac{\beta_{n}^{*}}{2} a_{n_{0}}
\end{gathered}
$$

with :

$$
a_{n_{0}} \# \frac{3}{2}\left(1-s_{n}^{2}\right) \Delta_{n}
$$

which formulae put in (27) do give expression (28). If $\left|\beta_{n}^{*}\right|$ is not very small, (29) gives $N$ quasi-classical solutions $u_{0, n}$ approximately given by (33), and it would seem that such a splitting contradicts expression (10) after which $u_{0}$ should be associated with a collective response of the conduction electrons.

However, uncoupled equations $(29,32)$ lead to numerical values of $Z_{\mathrm{s}}$ which remain in very good agreement (relative precision of a few $10^{-3}$ ) with those deduced from the exact coupled equations $(2,4)$, up to wavelengths about $20 \mu \mathrm{m}$ where $\left|\beta_{n}^{*}\right|$ approaches unity. It is shown in the following subsection that this worth noting point is due to the mathematical form of the surface impedance expression. 
3.4. WAVELENGTH DOMAIN OF VALIDITY OF THE E.M. UNCOUPLED ELECTRONS APPROXIMATION. - The above mentioned good agreement between exact and approximate uncoupled equations occurs in spite of an apparently unphysical splitting of the quasi-classical component in these latters. That may be understood if one develops the quasi-classical part $Y_{0}$ of the surface admittance to first order in $\beta_{n}$ or $\beta_{n}^{*}$ :

\section{* Exact equations.}

After (14), we have simply :

$$
Y_{0}=\frac{1}{\omega \mu_{0}} a_{0} u_{0}
$$

Neglecting the non diagonal components of the non local part of the electric field matrix (that has been justified in subsection 2.3), we get from (4a) :

$$
a_{0}=1-\sum_{p=1}^{N} a_{p} \# 1-\sum_{n=1}^{N} a_{n n}
$$

or after (20) :

$$
a_{0} \# 1-\sum_{n=1}^{N}\left(1-p_{n}\right) \frac{\beta_{n}}{2}
$$

After (10) and (11), the quasi-classical wavevector may be written as :

$$
u_{0} \# u_{c l}\left(1-\frac{1}{2} \sum_{n=1}^{N} \beta_{n}\right)
$$

It is then obtained :

$$
Y_{0} \# \frac{1}{Z_{c l}}\left[1-\sum_{n=1}^{N}\left(2-p_{n}\right) \frac{\beta_{n}}{2}\right] .
$$

\section{* Uncoupled equations.}

The contributions of the $N$ independent quasiclassical waves must be added, i.e. :

$$
Y_{0}=\frac{1}{\omega \mu_{0}} \sum_{n=1}^{N} a_{n_{0}} u_{0, n} .
$$

We get from (32) :

$$
a_{n_{0}} \# \frac{3}{2}\left(1-s_{n}^{2}\right) \Delta_{n}\left[1-\left(1-p_{n}\right) \frac{\beta_{n}^{*}}{2}\right] .
$$

Using (33), it is obtained :

$$
Y_{0} \# \frac{u_{c l}}{\omega \mu_{0}} \sum_{n=1}^{N} \frac{3}{2}\left(1-s_{n}^{2}\right) \Delta_{n}\left[1-\left(2-p_{n}\right) \frac{\beta_{n}^{*}}{2}\right] .
$$

Remembering that $\beta_{n}=\frac{3}{2}\left(1-s_{n}^{2}\right) \Delta_{n} \beta_{n}^{*}$ and assuming a sufficiently large value for $N$, we indeed retrieve expression (35).

Thus, it would seem that the uncoupled equations are valid in the relaxation region if :

$$
\left|\beta_{n}^{*}\right| \ll 1
$$

or, after (8), if :

$$
\left(\frac{v_{\mathrm{F}}}{c} \frac{\omega_{0}}{\omega}\right)^{2} \ll 1 .
$$

In fact, figure 1 exhibits a significant discrepancy between the curves obtained from the exact and uncoupled equations only if $\lambda \gtrsim 20 \mu \mathrm{m}$.

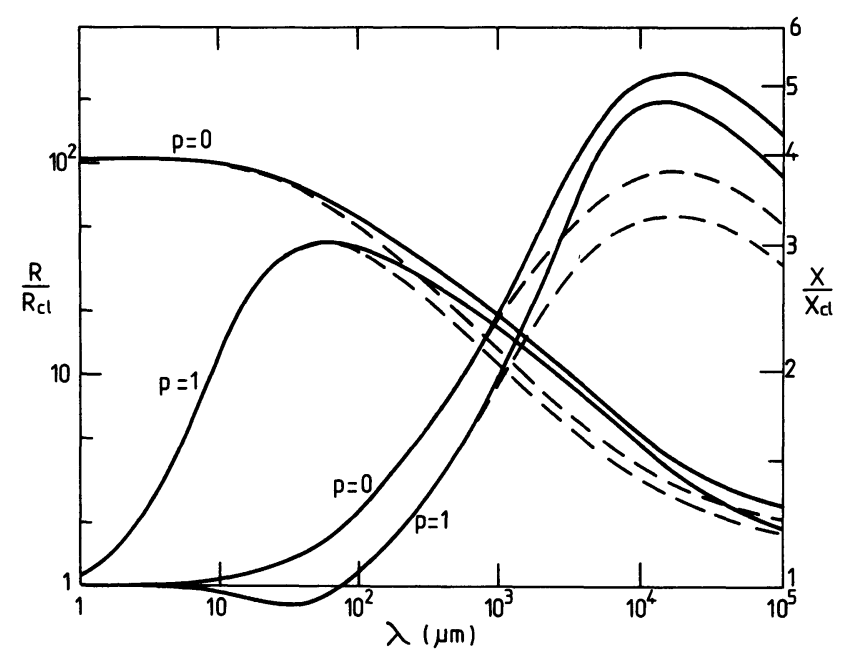

Fig. 1. - Surface impedance of copper. Comparison between the uncoupled electrons approximation (full curves) and Dingle's series (broken curves) for $l=5 \mu \mathrm{m}$ and $p=0$ and 1 (completely diffuse and specular scattering). The surface resistance and reactance are rapported to their classical values $R_{c l}$ and $X_{c l}$ obtained from the Drude theory.

This limiting value corresponds to $\left|\beta_{n}^{*}\right| \approx 1$; it implies therefore that uncoupled equations are accurate to powers of $\beta_{n}^{*}$ greater than unity.

Moreover, it has been found in reference [1] (inequality (11)) that the local theory is valid (more precisely that the electric field space variation is the classical exponential law) if :

$$
\frac{l}{\delta} \frac{\sqrt{2}}{\left(1+\omega^{2} \tau^{2}\right)^{3 / 2}} \ll 1
$$

with $\delta=\left(2 / \sigma \omega \mu_{0}\right)^{1 / 2}$ : classical skin depth expression in the microwave region.

In the relaxation region $(\omega \tau \gg 1)$, this condition becomes :

$$
\frac{v_{\mathrm{F}}}{c} \frac{\omega_{0}}{\omega} \ll 1 .
$$

It corresponds to $\left|\beta_{n}^{*}\right|^{1 / 2} \ll 1$ which is a more stringent condition than (36).

It may be finally concluded from the preceding considerations that the surface impedance may be computed according to three levels of approximation which are, by ascending degree of complexity : 
3.4.1 Visible and very near infrared $(\lambda \lesssim 1 \mu \mathrm{m})$. The condition $\left|\beta_{n}^{*}\right|^{1 / 2} \ll 1$ equivalent to Reuter and Sondheimer's inequality (9) of reference [2] is fulfilled. The classical local equations are valid, except in the vicinity of the surface $(z \approx l)$ if $p \neq 1 . Z_{\mathrm{s}}$ is then simply expressed by (23). It differs from its classical value only by an additional temperature independent surface assisted absorption term included in its real part.

An eventually anisotropic specularity parameter $p(s)$ may be taken into account by means of integral (24).

3.4.2 Weak anomalous skin effect region $(1 \mu \mathrm{m}<\lambda$ $\lesssim 20 \mu \mathrm{m})$. - The corresponding conditions are :

$$
\omega \tau>1 \quad \text { and } \quad\left|\beta_{n}^{*}\right| \lesssim 1 .
$$

Non local equations must be used but the electrons may be treated independently one from each other. In practice, the calculations are made on $N$ independent sets $s_{n}$ of electrons by using uncoupled equations $(29,32)$. The surface admittance is given by :

$$
Y_{\mathrm{s}}=\frac{1}{\omega \mu_{0}} \sum_{n=1}^{N}\left(a_{n_{0}} u_{0, n}+a_{n n} u_{n}\right) .
$$

3.4.3 Anomalous skin effect region $(\lambda>20 \mathrm{~nm})$. No approximation can be made and one must solve the exact coupled equations $(2,4)$ and compute $Z_{\mathrm{s}}$ by using (14).

\section{Numerical results.}

Since the aim of our computations is to check the accuracy of the approximate non local uncoupled equations, we have put $\varepsilon_{\mathrm{r}}=0$ for simplicity.

In the isotropic approximation, one needs then only two intrinsic constants of the metal for computing the surface impedance. We have used hereafter the datas of copper $[11,12]$ :

$$
\frac{\sigma}{l}=1.515 \times 10^{15} \Omega^{-1} \mathrm{~m}^{-2}
$$

and :

$$
v_{\mathrm{F}}=1.59 \times 10^{6} \mathrm{~m} \mathrm{~s}^{-1} .
$$

In addition, it must be noticed that in the wavelength domain of interest, Holstein's bulk absorption process [13] does not permit very large electron mean free path. Following Mc Kay and Rayne [5] who have derived a simple collision frequency expression from the quantum-mechanical treatment of Holstein [14], it is found that, in normal noble metals, the electron mean free path maximum value is only of a few $10^{-7} \mathrm{~m}$. Thus, such curves as those of figure 1 for $l=5 \mu \mathrm{m}$ (an approximate value at $\omega=0$ and liquid helium temperature [11]) must be considered simply as mathematical tests of the equations.

4.1. VALUES OF $Z_{\mathrm{s}}$ IN WEAK ANOMALOUS SKIN EFFECT CONDITIONS. - In figure 1 are plotted the real and

Table II. - Comparison of surface impedance values obtained from Dingle's series, approximate uncoupled equations $(N=20)$ and exact coupled equations $(N=6)$ in the limit cases of completely specular $(p=1)$ and completely diffuse $(p=0)$ electron-surface scattering. The electron mean free path is $l=0.1 \mu \mathrm{m}$. One complex value is represented by its real (upper number) and imaginary (lower number) parts rapported to their classical values.

\begin{tabular}{|c|l|l|l||l|l|l|}
\hline \multirow{2}{*}{$\lambda(\mu \mathrm{m})$} & \multicolumn{3}{|c||}{$p=1$} & \multicolumn{3}{c|}{$p=0$} \\
\cline { 2 - 7 } & \multicolumn{1}{|c|}{ Dingle } & $\begin{array}{c}\text { appr. } \\
N=20\end{array}$ & $\begin{array}{c}\text { exact } \\
N=6\end{array}$ & Dingle & $\begin{array}{c}\text { appr. } \\
N=20\end{array}$ & $\begin{array}{c}\text { exact } \\
N=6\end{array}$ \\
\hline \multirow{2}{*}{2} & 1.016 & 1.018 & 1.016 & 3.056 & 3.059 & 3.072 \\
& 0.999 & 0.999 & 0.997 & 1.001 & 1.001 & 0.999 \\
\hline \multirow{2}{*}{5} & 1.091 & 1.090 & 1.092 & 3.021 & 3.019 & 3.039 \\
& 0.995 & 0.995 & $0.99 ?$ & 1.005 & 1.005 & 1.004 \\
\hline \multirow{2}{*}{10} & $1.278\left(^{*}\right)$ & 1.275 & 1.282 & 2.914 & 2.910 & 2.932 \\
& 0.987 & 0.986 & 0.985 & 1.019 & 1.018 & 1.017 \\
\hline \multirow{2}{*}{20} & $1.553\left(^{*}\right)$ & 1.547 & 1.557 & $2.794\left(^{* *}\right)$ & 2.654 & 2.660 \\
& 0.981 & 0.980 & 0.980 & 1.049 & 1.055 & 1.056 \\
\hline \multirow{2}{*}{50} & $1.589\left(^{*}\right)$ & 1.610 & 1.590 & 2.084 & 2.111 & 2.081 \\
& 1.033 & 1.030 & 1.032 & 1.169 & 1.166 & 1.168 \\
\hline
\end{tabular}
of $\xi$.

$\left.{ }^{*}\right)$ Exact value obtained from direct integration for $p=1$ in the region where $|\xi|$ is about 0.8 (see Ref. [6] for definition

$(* *)$ Not reliable value because it has been obtained from Dingle's series for $|\xi| \approx 0.8$. 
imaginary parts of the surface impedance expressed as their ratio to their classical value, versus wavelength.

The mean free path $l=5 \mu \mathrm{m}$ is far greater than the one expected at room temperature $(37 \mathrm{~nm}$ at zero frequency [15]).

The continuous and broken curves have been computed respectively by means of uncoupled equations $(29,32,37)$ and Dingle's series [6, 16].

The agreement is excellent at wavelengths shorter than $20 \mu \mathrm{m}$. Beyond this limit, the discrepancy cannot be reduced, as this is the case for the exact discretized coupled equations, by increasing the number $N$ of components.

Table II indicates that the relative precision to be expected from the uncoupled equations is about $10^{-3}$ for $\lambda<10 \mu \mathrm{m}$.

This is a better accuracy than the one obtained from the exact discretized coupled equations for the maximum number of components $N=6$ allowed by our algorithms. We have represented in figure 2 the electric field amplitude space variation inside the metal.

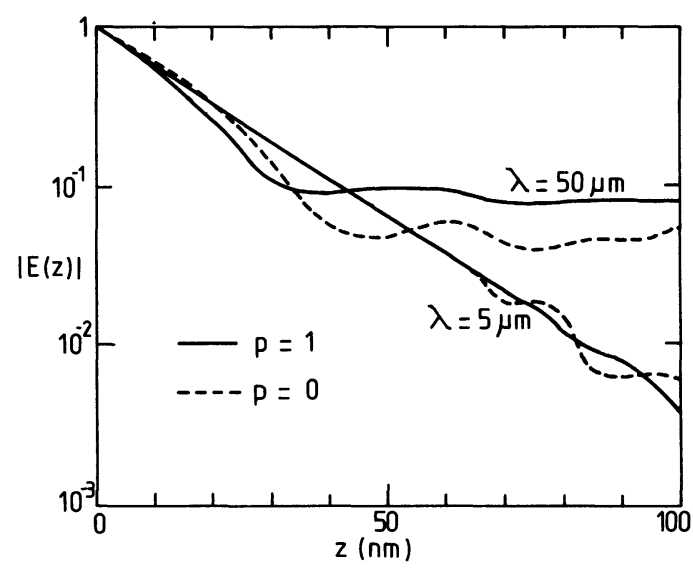

Fig. 2. - Electric field amplitude space variation inside the metal for $l=5 \mu \mathrm{m}$. The exact coupled equations have been used with $N=6$. The amplitude at $z=0$ is assumed to be unity. The classical Drude theory gives a straight line (exponential law in linear scales) which cannot be distinguished from the curves plotted for $\lambda=5 \mu \mathrm{m}$ near the metal surface $(z<60 \mathrm{~nm})$. At longer wavelengths, the field decreases first more rapidly near the surface $(z<50 \mathrm{~nm})$ and remains then nearly constant at deeper points. The oscillations of the curves at $z>50 \mathrm{~nm}$ are due to the relatively low value $N=6$ allowed by the exact algorithms.

It has been computed by using the exact discrete equations $(2,4)$. The curves for $\lambda=5 \mu \mathrm{m}$ cannot be distinguished from the straight line corresponding to the classical curve when $z<60 \mathrm{~nm}$. The oscillations that appear at greater $z$ are due to the finite number $N=6$ of partial waves. In the wavelength domain where uncoupled equations are not valid, the curves diverge significantly from the classical one as shown for $\lambda=50 \mu \mathrm{m}$. The "tail» which characterizes the anomalous skin effect appears clearly in this latter case, at $z$ deeper than $30 \mathrm{~nm}$. This figure illustrates the point mentioned by Pippard [17] according to which the electric field cannot be approximated, even roughly, by an exponential solution beyond a definite wavelength limit.

\subsection{CONDUCTION ELECTRONS EFFECTIVENESS FUNC-} TION. - In most experiments the quantity of interest is the absorptance; thus, we shall consider that an electron is the more effective as the E.M. wave energy loss after a collision is more important.

Under normal incidence of the external wave, the absorptance is given by :

$$
\mathcal{A} \# 4 \frac{R_{\mathrm{s}}}{Z_{0}} \text {. }
$$

This expression is valid if the surface resistance $R_{\mathrm{s}}$ is small compared with the free space impedance $Z_{0}$. Using definition (15a) of the partial surface admittances $Y_{n}$, the relative contribution of electrons $s_{n}$ may thus be written as :

$$
\frac{\mathcal{A}_{n}}{\mathcal{A}} \# \frac{\operatorname{Re}\left\{Y_{n}\right\}}{\operatorname{Re}\left\{Y_{\mathrm{s}}\right\}}=F_{n} \Delta_{n}
$$

where $\mathcal{A}_{n}$ is the absorptance due to electrons $\left[s_{n}-\frac{\Delta_{n}}{2}, s_{n}+\frac{\Delta_{n}}{2}\right]$. We have defined in this way a discrete effectiveness function $F_{n}$ that may be related for $N \rightarrow \infty$ to the continuous one $F(s)$ by :

$$
F_{n}=F\left(s_{n}\right) \text {. }
$$

In pratice, this relation holds even when $N$ is not very large, the discrepancy being only a few percents for $N=4$ and less than one percent for $N=6$.

Consequently, we have plotted the curves by using the formula :

$$
F\left(s_{n}\right) \# \frac{1}{\Delta_{n}} \frac{\mathcal{R e}\left\{Y_{n}\right\}}{\operatorname{Re}\left\{Y_{\mathrm{s}}\right\}}
$$

As $Y_{s}=\sum_{n=1}^{N} Y_{n}$, we have the additional condition

$$
\int_{0}^{1} F(s) \mathrm{d} s=1
$$

The quasi-classical and non local contributions may be separated in (38) and the total effectiveness function expressed as :

$$
F=F_{0}+f
$$

where $F_{0}$ and $f$ are respectively the quasi-classical and non local parts.

In the exact discrete model, these contributions will be expressed after (14) by :

$$
F_{0}=\frac{1}{D} \mathcal{R e}\left\{a_{n_{0}} u_{0}\right\}
$$


and :

$$
f=\frac{1}{D} \operatorname{Re}\left\{\sum_{p=1}^{N} a_{n p} u_{p}\right\} .
$$

In the uncoupled electrons model, they will be expressed, after (37) by :

$$
F_{0}=\frac{1}{D} \operatorname{Re}\left\{a_{n_{0}} u_{0, n}\right\}
$$

and :

$$
f=\frac{1}{D} \operatorname{Re}\left\{a_{n n} u_{n}\right\}
$$

with :

$$
D=\omega \mu_{0} \Delta_{n} \operatorname{Re}\left\{Y_{\mathrm{s}}\right\} .
$$

We have plotted in figure 3 effectiveness functions computed with the exact coupled equations for $N=6$ and $l=0.1 \mu \mathrm{m}$. The continuous curves correspond to an anisotropic surface scattering parameter expressed by the following Soffer formula [18] :

$$
p(s)=\exp \left[-\left(4 \pi h s / \lambda_{b}\right)^{2}\right]
$$

in which $\lambda_{\mathrm{b}}$ and $h$ are respectively the De Broglie electron wavelength on the Fermi surface and the rms height of the metal surface roughness.

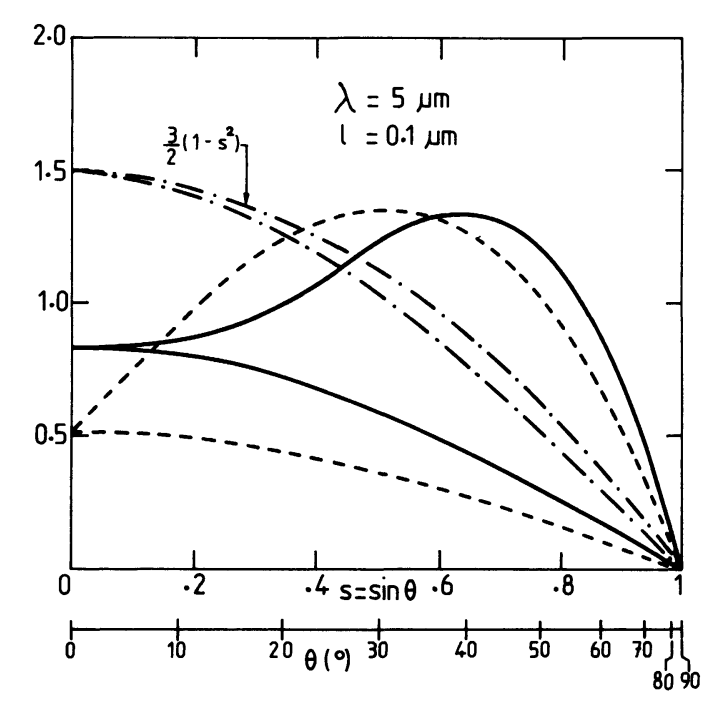

Fig. 3. - Effectiveness functions computed with the exact coupled equations for $N=6, \lambda=5 \mu \mathrm{m}$ and $l=0.1 \mu \mathrm{m}$ in the completely specular $(-., .-)$ and completely diffuse $\left(--_{-}\right)$electron-surface scattering cases. The continuous curves have been plotted for the anisotropic Soffer specularity parameter expression. The practical formula which has been used here for copper is :

$$
p(s)=\exp \left(-7.55 . h^{2} s^{2}\right)
$$

where $h$ is the rms height of the surface roughness in angstroms (it has been taken equal to $0.5 \AA$; that corresponds after (24) to $\langle p\rangle=0.6$ ). For a given speculariry parameter, the upper curve is the total effectiveness function as defined by (38) whereas the lower one is the quasi-classical contribution expressed by (39 a).
For a given specularity parameter function or value, the upper curve represents the total effectiveness function whilst the lower one is the quasi-classical contribution as defined by (39a). The most striking features of these curves are that they vary smoothly and exhibit a maximum which is not located at $s=0$ when electron-surface scattering is not completely specular. The continuous curves indicate that with the Soffer model, the electrons which move at grazing angles act quasi-classically (no surface absorption).

In figure 4 are compared the preceding curves (broken lines) with those computed by means of the uncoupled equations (continuous lines).

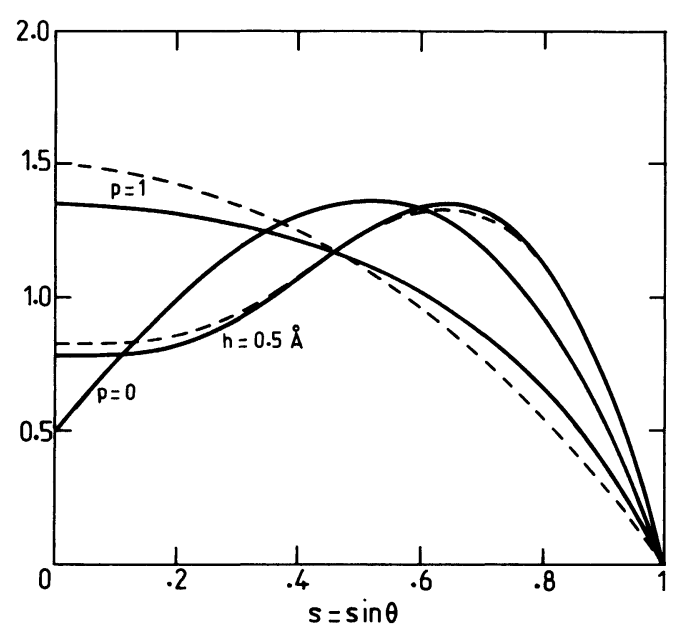

Fig. 4. - Comparison between the exact total effectiveness functions already plotted in figure 3 (dashed line) and those obtained from the uncoupled equations (continuous line). The discrepancy for $p=1$ decreases at shorter wavelengths; it does not affect the total surface impedance value which is, as indicates table II, practically the same in both cases.

The agreement, which is excellent in the completely diffuse scattering case, is not as good when $p=1$ though a coincidence of $10^{-3}$ between the corresponding impedance values has been obtained (see Table II).

This discrepancy decreases at higher frequencies; it usually has a negligible effect on the total admittance, specially when $p$ approaches to zero.

At longer wavelengths $(\lambda>10 \mu \mathrm{m})$, an error on the total admittance will appear if electron-surface scattering is essentially specular and if the relaxation time is a rapidly varying function of $s$, as that may be the case in thin polycrystalline films as modelized for example by Mayadas and Shatzkes [19].

4.3. SPeCUlarity Weighting FUNCTION. - It has been found in [1] that the surface resistance may be computed precisely for any anisotropic specularity parameter, by means of the relation :

$$
R_{\mathrm{s}}=\langle p\rangle R_{1}+(1-\langle p\rangle) R_{0}
$$


with :

$$
\langle p\rangle=\sum_{n=1}^{N} W_{n} p_{n} \quad \text { in the discrete case }
$$

or :

$$
\langle p\rangle=\int_{0}^{1} w(s) p(s) \mathrm{d} s \text { in the continuous case. }
$$

$R_{0}$ and $R_{1}$ are the surface resistance values calculated respectively for a completely diffuse and specular scattering.

$W_{n}$ and $w(s)$ are the discrete and continuous specularity weighting functions.

The former may be easily calculated with the expression :

$$
W_{n}=\frac{R_{0}-R_{n}}{R_{0}-R_{1}}
$$

Where $R_{n}$ is the value of $R_{\mathrm{s}}$ computed for the specularity distribution $p_{m}=\delta_{n m}, m=1$ to $N$. $w(s)$ will be deduced from $W_{n}$ in an analogous manner as the one used for the preceding function $F(s)$, by the formula :

$$
w\left(s_{n}\right) \# W_{n} / \Delta_{n} .
$$

In the relaxation region, it is given after (24) by :

$$
w(s)=4 s\left(1-s^{2}\right) .
$$

This expression has been plotted in continuous line in figure 5. It gives identical results to those deduced from the exact discrete coupled equations by means of (41) (relative precision better than one percent for $N=6)$ when $\lambda<2 \mu \mathrm{m}$. The maximum that occurs at $s=1 / \sqrt{3}$ indicates that the electrons which move at angles $\theta \approx 35^{\circ}$ are the more sensitive to surface scattering effects.

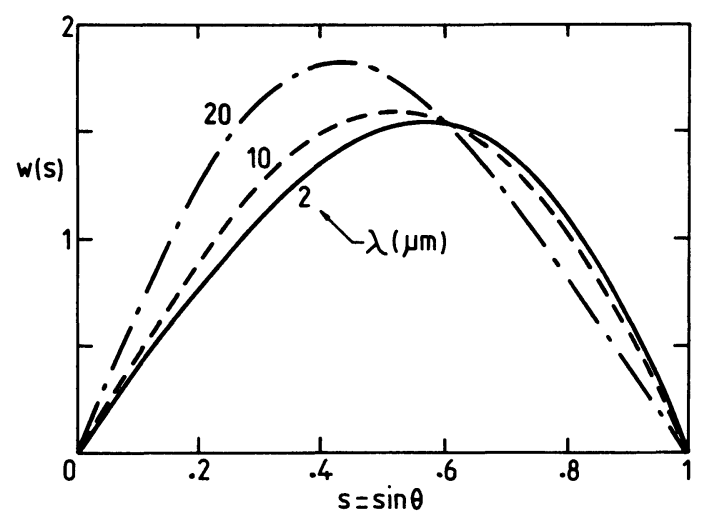

Fig. 5. - Specularity weighting functions (defined in text in subsection 4.3) for $l=5 \mu \mathrm{m}$.

Identical curves (within a few $10^{-3}$ ) are obtained from both exact and uncoupled discrete equations for $N=6$.

The full curve plotted for $\lambda=2 \mu \mathrm{m}$ coincides with expression (42) valid in the near infrared and visible domains.
If the wavelength is increased, this maximum is shifted towards smaller $\theta$.

In the submillimetric and microwave domains where the surface impedance is nearly insensitive to electron-surface scattering conditions, the knowledge of $w(s)$ becomes of very little interest.

The coincidence with the same curves obtained from the uncoupled equations is very good, about $10^{-3}$ for the limiting value $\lambda=20 \mu \mathrm{m}$.

Thus, the uncoupled electrons approximation allows accurate predictions about the effects of an anisotropic specularity parameter.

\subsection{WAVELENGTH DEPENDENCE OF THE EFFECTIVENESS} FUNCTION. - We have plotted in figure 6 the angular effectiveness functions $G(\theta)$ which are related to $F(s)$ by :

$$
G(\theta)=F(s=\sin \theta) \cos \theta
$$

because it is assumed that :

$$
\int_{0}^{\pi / 2} G(\theta) \mathrm{d} \theta=1
$$

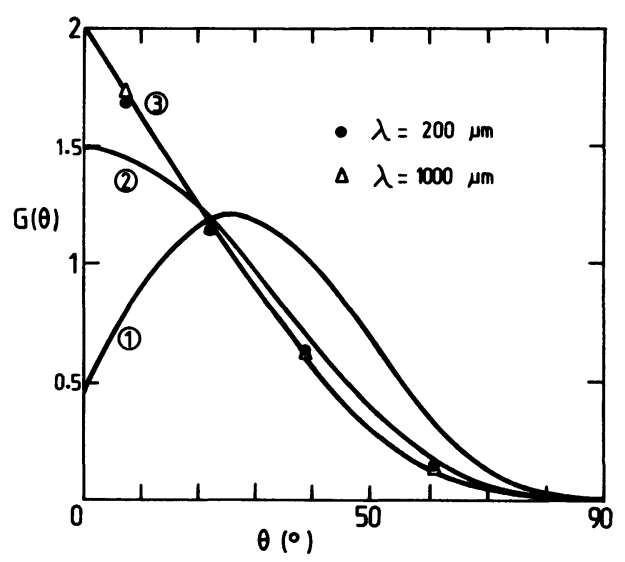

Fig. 6. - Angular dependence of the effectiveness functions plotted for $l=0.1 \mu \mathrm{m}$ in some extreme situations.

1. - Near infrared and completely diffuse surface scattering. Formula (44) gives for copper :

$$
G(\theta)=0.49(1+5.5 \sin \theta) \cos ^{3} \theta
$$

2. - Completely specular surface scattering. One gets after (43) :

$$
G(\theta)=1.5 \cos ^{3} \theta
$$

This relation is not wavelength dependent.

3. - Submillimetric domain and completely diffuse surface scattering. The theoretical points computed for $\lambda=200$ and $1000 \mu \mathrm{m}$ are well fitted with expression (45); that corresponds to :

$$
G(\theta)=2(1-\sin \theta) \cos \theta
$$

It appears that in extreme anomalous skin effect conditions, the effectiveness functions are not frequency dependent but depend strongly on the scattering boundary conditions for electrons moving at grazing angles. 
An important point deduced from numerical results of the exact coupled equations is that the effectiveness function is not wavelength dependent in the completely specular scattering case. It remains identical to the high frequency limit (curve 2 of Fig. 6) expressed, after definition (39) and relations of subsections 2.4 and 3.1, by :

$$
F(s)=\frac{3}{2}\left(1-s^{2}\right)
$$

This expression is valid even in extreme anomalous skin effect conditions and may be physically interpreted as a consequence of the above mentioned tail in the electric field profile (see Fig. 2 for $\lambda=50 \mu \mathrm{m}$ ) allowing significant interaction with electrons which does not move at grazing angles.

On the other hand, when electron surface scattering is completely diffuse, the near infrared effectiveness function is given, after subsections 2.4 and 3.1 , by :

$$
F(s)=\frac{3}{2}\left(1-s^{2}\right)\left(1+\frac{\omega_{0}}{c} l s\right) / K
$$

where $K$ is a normalization constant.

The corresponding curve 1 of figure 6 is very different in shape from curve 3 which has been fitted to theoretical points for $\lambda=200$ and $1000 \mu \mathrm{m}$.

This latter may be fairly approximated by the linear expression of $s$ :

$$
F(s)=2(1-s)
$$

Thus, the assumption of Pippard [20] according to which the only effective electrons are those which move in directions nearly parallel with the surface is valid if the scattering is not completely specular.

From a physical point of view, it seems that in extreme anomalous skin effect conditions, the surface assisted absorption associated with electrons moving at grazing angles becomes rapidly predominant as the scattering tends to be diffuse.

It follows that, if as predicted by most models $[18,21]$ electron-surface scattering is specular as $\theta \rightarrow 0$, the effectiveness function will present a sharp maximum which will not be located exactly at $\theta=0$.

This phenomenon may introduce errors in measurements such as those of Pippard [22] for the determination of the Fermi surface of copper.

\section{Conclusion.}

Starting from a discrete $N$-directional model for the anomalous skin effect, it has been shown that electronelectron EM coupling becomes very small in the relaxation region and thus, the Maxwell-Boltzmann propagation equation could be simplified in such a way that each conduction electron may be treated independently from each other (Eq. (30)). Consequently, a similar model to the $N$-directional preceding one is proposed in which the equations of the anomalous skin effect theory are solved for $N$ independent sets of electrons moving at angles $\theta_{n}$ with respect to the metal surface.

The surface impedance is then expressed as the reciprocal sum of the corresponding partial admittances $Y_{n}$.

It has been proved that both methods are equivalent when the parameter $\beta^{*}$ defined in subsection 3.2 tends to zero. Moreover, if the condition $\left|\beta^{*}\right|^{1 / 2} \ll 1$ is fulfilled, the simple surface impedance expression(23) is valid.

In the wavelength domain where the above condition is not verified but if $\left|\beta^{*}\right|<1$, it has been found that the uncoupled electrons approximation gives very good numerical results for any mean free path value, provided that $\omega \tau>1$. That corresponds to the weak anomalous skin effect domain where the electric field divergence from the classical exponential law is rather weak but not negligible if a relative precision about $10^{-3}$ is required.

It has come recently to the knowledge of the author that Pippard has used the same approach in 1947 [20] for a qualitative analysis of the anomalous skin effect in the microwave region. However, he did not propose a numerical solution of his discrete equations and could not then compute the electric field profile inside the metal, nor the effectiveness function.

In a recent work of the present author, the uncoupled electrons approximation has revealed to be a useful tool for the interpretation of optical measurements on very thin polycrystalline films in which two kinds of anisotropy must be taken into account : one for the relaxation time $[19,30]$ and the other for the specularity parameters at both surfaces $[18,21]$.

This approximate but nevertheless accurate model allows the use of large values of $N$ and therefore, an exact computation of the bulk relaxation time when the film morphology is known.

It is then possible to interpret the wavelength dependence of the bulk relaxation time in the framework of Holstein theory [13, 14] and estimate the order of magnitude of e-e collisions.

Such computations are very difficult to make with the initial exact discrete equations because the algorithms become untractable when $N$ is greater than about ten. Approximate solutions of the anomalous skin effect equations have been presented elsewhere [23-29] but anisotropy cannot be easily introduced in most of them.

In addition, a simple exponential space variation is sometimes assumed for the electric field [27, 29] which forbids a very accurate calculation of the surface impedance in the weak anomalous skin effect domain.

From a practical point of view, the present method is easy to implement on a digital computer since the same relatively simple equations ((29) and (32) in the semi-infinite case) are to be solved $N$ times. 


\section{References}

[1] Dudek, J. C., Rev. Phys. Appl. 19 (1984) 573.

[2] Reuter, G. E. H., Sondheimer, E. H., Proc. R. Soc. A 195 (1948) 336.

[3] Dingle, R. B., Appl. Sci. Res. B 3 (1953) 69.

[4] Holstein, T., Phys. Rev. 88 (1952) 1427.

[5] Mc Kay, J. A., RaYne, J. A., Phys. Rev. B 13 (1976) 673.

[6] Dingle, R. B., Physica 19 (1953) 311.

[7] Hodgson, J. N., J. Phys. Chem. Solids 29 (1968) 2175.

[8] Theye, M. L., Phys. Rev. B 2 (1970) 3060.

[9] Schlesinger, Z., Sievers, A. J., Solid State Commun. 43 (1982) 671.

[10] Beach, R. T., Christy, R. W., Phys. Rev. B 16 (1977) 5277.

[11] Peterseim, J., Thummes, G., Mende, H. H., Phys. Status Solidi 59 (1980) K 25.

[12] Kittel, C., Introduction à la physique de l'état solide (3e édition, Dunod, 1972).

[13] Holstein, T., Phys. Rev. 96 (1954) 535.

[14] Holstein, T., Ann. Phys. 29 (1964) 410.

[15] White, G. K. and Woods, S. B., Philos. Trans. R. Soc. A 251 (1959) 273.
[16] Dudek, J. C., Rev. Phys. Appl. 19 (1984) 287.

[17] Pippard, A. B., Reuter, G. E. H. and Sondheimer, E. H., Phys. Rev. 73 (1948) 920.

[18] SOFfer, S. B., J. Appl. Phys. 38 (1967) 1710.

[19] Mayadas, A. F. and Shatzkes, M., Phys. Rev. B 1 (1970) 1382.

[20] Pippard, A. B., Proc. R. Soc. A 191 (1947) 385.

[21] Greene, R. F., Phys. Rev. 141 (1966) 690.

[22] Pippard, A. B., Philos. Trans. R. Soc. 250 (1957) 325.

[23] Dingle, R. B., Physica XIX (1953) 1187.

[24] Drangeid, K. E. and Sommerhalder, R., Helv. Phys. Acta 48 (1975) 561.

[25] Dimmich, R. and Warkusz, F., Phys. Status Solid. 72 (1982) 117.

[26] Gilberd, P. W., J. Phys. F. : Met. Phys. 12 (1982) 1845.

[27] Jauho, A. P. and Smith, H., J. Phys. F. : Met. Phys. 15 (1985) 1951.

[28] Szczyrbowski, J., DryzeK, J. and Czapla, A., Thin Solid Films 112 (1984) 175.

[29] Szczyrbowski, J., Schmalzbauer, K. and Hoffman, H., Phys. Rev. B 32 (1985) 763.

[30] DudeK, J. C., Thin Solid Films 137 (1986) 11. 